\title{
Las enzimas limpiadoras
}

Carmen-Lisset Flores y Carlos Gancedo

Instituto de Investigaciones Biomédicas "Alberto Sols" CSIC-UAM. 28029 Madrid

clflores@iib.uam.es cgancedo@iib.uam.es
El funcionamiento correcto del metabolismo es fundamental para la vida de los organismos. Los libros de bioquímica han presentado una imagen del metabolismo que, si bien facilita su estudio, en ocasiones no refleja la complejidad de la red metabólica. En general el metabolismo se muestra como una maquinaria perfecta en la que las enzimas de las distintas vías aparecen como los actores principales de la gran función de la vida. Sin embargo, a veces, esas enzimas generan productos impropios, bien porque la reacción enzimática tome un camino "equivocado" desde el complejo activado o porque la enzima actúe sobre un substrato no adecuado. Por otra parte, algunos metabolitos pueden sufrir transformaciones no enzimáticas y generar productos no deseados. Esos productos pueden ser tóxicos y hay que eliminarlos 0 mantenerlos a bajas concentraciones. En este breve artículo queremos llamar la atención sobre los actores secundarios del metabolismo, las enzimas limpiadoras que permiten a las células llevar una vida normal. Nos provoca a ello un reciente artículo de revisión sobre algunas de esas enzimas, ignoradas

Figura 1. Ejemplos de producción de metabolitos erróneos.- Los metabolitos erróneos se muestran en negrita. Las enzimas limpiadoras que los eliminan se mencionan en el texto.

A. La gliceraldehído 3-P deshidrogenasa puede actuar sobre la eritrosa 4-P iniciando la formación de eritronato 4-P un potente inhibidor de la 6 P-gluconato deshidrogenasa. A la izquierda se muestra la reacción metabólica principal.

B. Formación de NADHX a partir de NAD. Este producto se forma por la acción de la gliceraldehído

3-P deshidrogenasa o espontáneamente y es un inhibidor de deshidrogenasas dependientes de NAD.

C. La acción de la hexokinasa sobre el 1,5 anhidroglucitol, produce 1,5 anhidroglucitol 6-P que es un inhibidor de la propia enzima. A la izquierda aparece la reacción metabólica de la enzima.
A
Gliceraldehído 3-P<smiles>O=CC(I)CO[OH2+]</smiles>

$\mathrm{OH}$

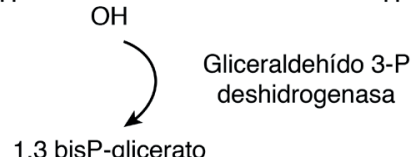

1,3 bisP-glicerato

6P-gluconato deshidrogenasa

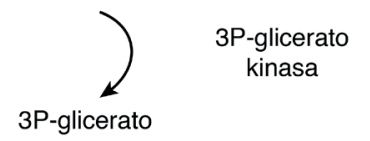

Eritrosa 4-P<smiles>O=CC(I)C(I)CO</smiles>

$\mathrm{OH} \mathrm{OH}$
1,4 bisP-eritronato<smiles>CCCCCCC</smiles>

4P-eritronato

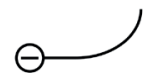

B

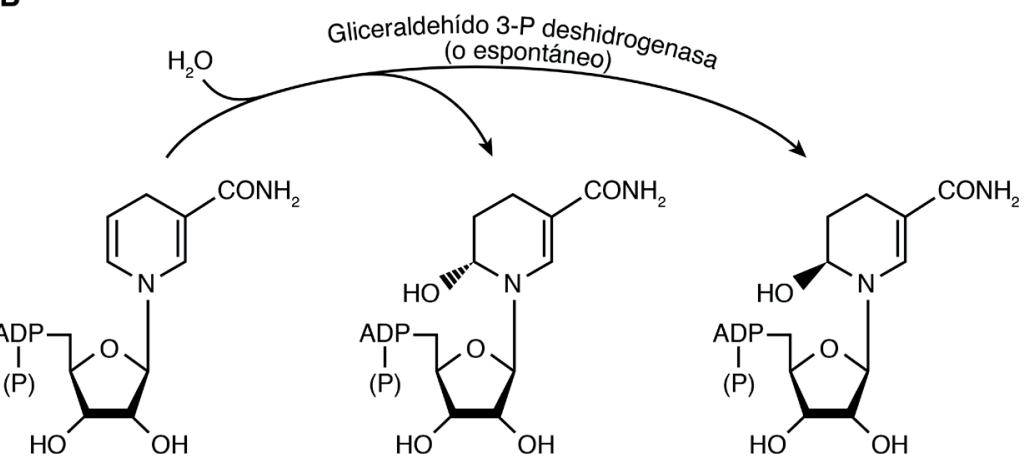

$\mathrm{NADH}$

(R)-NADHX

(S)-NADHX

C

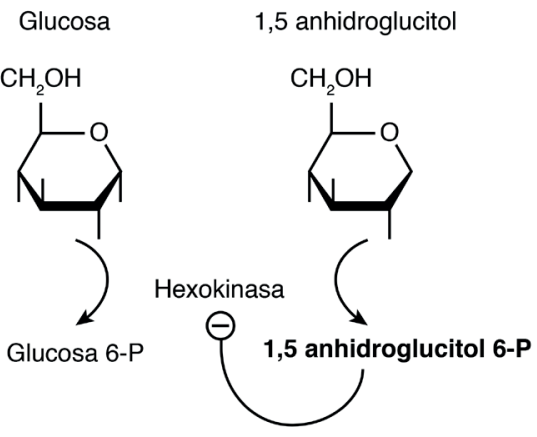


o desconocidas hasta hace poco, publicado por el grupo de Emile van Schaftingen del Instituto De Duve de la Universidad Católica de Louvain-la Neuve (Bélgica) (Bommer et al., 2020). Ese tipo de enzimas limpiadoras se ha descrito en eucariotas pluricelulares, levaduras y bacterias, sugiriendo que son fundamentales para distintas formas de vida. En esta rápida pincelada ilustrativa consideraremos solo algunos casos representativos, remitiendo para más información a la revisión mencionada y a los artículos allí citados.

La gliceraldehído-3-P deshidrogenasa, una abundante enzima glicolítica (alrededor de 170000 moléculas por célula de levadura), es capaz de actuar marginalmente sobre eritrosa 4-P produciendo, conjuntamente con la 3P-glicerato kinasa, 4 P-eritronato que inhibe fuertemente la 6 P-gluconato deshidrogenasa y por tanto bloquea la vía de las pentosas fosfato (Fig 1A). Collard et al. (2016) mostraron que en mamíferos existe una enzima, la P-glicolato fosfatasa, capaz de hidrolizar el metabolito espúreo. En levadura se había descrito una fosfatasa, Pho13, con gran especificidad para p-nitrofenil-P cuya función fisiológica era desconocida. Posteriormente se describió la actividad de esta fosfatasa frente a formas fosforiladas de la histona $2 \mathrm{~A}$ y de caseína, sugiriendo un papel de Pho13 en procesos de fosforilación reversible de proteínas. Pues bien, Pho13 ha resultado ser la enzima que, en levadura, "limpia" el 4 P-eritronato. En mutantes con una deleción de PH013 aumenta la expresión de genes codificantes de enzimas implicadas en la vía de las pentosas fosfato (Collard et al. 2016)) lo que permite compensar el efecto inhibidor del 4-P-eritronato.

También Collard et al (2016) mostraron que una actividad marginal de otra enzima glicolítica clave, la piruvato kinasa, producía en mamíferos 2 P-L-lactato, que resulta ser un inhibidor de la enzima que genera fructosa-2,6-bisP, el más potente activador de la fosfofructokinasa. Por tanto, la acumulación del 2 P-L-lactato provocaría una inhibición de la glicolisis; la misma P-glicolato fosfatasa que hidroliza el 4 P-eritronato, se encarga de eliminar el 2 P-L-lactato evitando el colapso de la glicolisis.

Otro caso que implica una actividad marginal de la gliceraldehído-3-P deshidrogena- sa es la producción de una forma de NADH con un grupo hidroxilo en C6 (NADHX) en las configuraciones epiméricas $R \circ S$ (Fig.1B). Esas formas no funcionan como coenzima en las reacciones enzimáticas e inhiben las deshidrogenasas dependientes de NAD. La forma $S$ es recuperada mediante la acción de una deshidratasa que consume ATP; para recuperar la forma $R$ se requiere adicionalmente una epimerasa que transforma la configuración $R$ en $S$. El NADHX se forma también espontáneamente y lo mismo sucede con la forma NADPHX. El mismo sistema de reparación actúa sobre las formas modificadas de las dos coenzimas y se ha identificado en todos los dominios de la vida. Su deficiencia causa problemas neurodegenerativos letales en mamíferos (Van Bergen et al. 2019, Becker-Kettern et al. 2018), depleción de la reserva de serina en levaduras (Becker-Kettern et al. 2018), o dificultad en la adaptación a estrés en Bacillus subtilis (Petrovova et al. 2014). Curiosamente la carencia de la deshidratasa no parece causar problemas en plantas (Colinas et al. 2014). Todavía faltan conocimientos para explicar cómo la ausencia del mismo sistema limpiador produce efectos diferentes en distintos organismos. Como se ha apuntado en otro contexto "in biology, details matter a lot, and we don't understand the details" (Silver et al. 2014)

El último caso que mencionaremos es el debido a la producción de un metabolito tóxico resultante del uso de un substrato erróneo por la hexokinasa. La hexokinasa, que inicia la glicolisis fosforilando glucosa a glucosa 6-P, fosforila también análogos de la glucosa. Sols y Crane mostraron hace más de cincuenta años (Sols y Crane 1954) que uno de esos análogos era el anhidroglucitol (Figura 1 C), un azúcar que se forma en la degradación de glicanos y que se encuentra en numerosos alimentos (Yamanouchi et al 1992). Pues bien, el producto de esa fosforilación, el anhidroglucitol 6-P es un fuerte inhibidor de la propia hexokinasa (Sols y Crane 1954). En mamíferos se ha encontrado que una acumulación de anhidroglucitol-6P causa neutropenia. ¿Cómo se evita normalmente que ese compuesto se acumule? Hay dos proteínas cuya acción secuencial se encarga de eliminarlo: el transportador de glucosa 6-P al retículo endoplásmico, que también transporta anhidroglucitol 6-P, y una fosfata- sa que convierte éste en anhidroglucitol que se excreta en orina. Una deficiencia en alguna de esas proteínas causa la acumulación del anhidroglucitol-6P que, al inhibir la hexokinasa en los neutrófilos dependientes de la glicolisis para la obtención de ATP, origina el problema mencionado (Veiga-da-Cunha et al. 2019).

Una pregunta interesante es la de si, a lo largo de la evolución, algún producto erróneo podría haber sido reclutado para desempeñar alguna función, convirtiéndose así en un metabolito más. Un ejemplo de esto podría ser el caso del diadenosin tetrafosfato (Ap4A). Este compuesto, un dinucleótido formado por dos adenosinas unidas en enlace 5'-5'por cuatro fosfatos, es producido por las aminoacil-tRNA sintetasas cuando no está presente el correspondiente tRNA. Se ha encontrado en numerosos organismos (Zamecnik, 1983) y se le ha propuesto como una alarmona. Sin embargo, un estudio en el que se examinan cuidadosamente una serie de criterios para ser calificado como tal parece demostrar que este no es el caso en Escherichia coli (Despotović et al. 2017). En contraste, recientemente se ha sugerido su posible papel como regulador en mastocitos (Yu et al. 2019). Por ello la idea general de reclutar un subproducto para una función sigue siendo atractiva ya que los caminos de la evolución son impredecibles.

Lo expuesto muestra que, en contra de opiniones extendidas, todavía ignoramos bastante sobre el metabolismo y que como el gran enzimólogo Alberto Sols decía a veces, "las enzimas son específicas, pero menos". Quizá esta llamada de atención sobre las enzimas limpiadoras - a las que el grupo de Van Schaftingen denomina enzimas reparadoras del metabolismo- despierte interés para identificar nuevas enzimas de este tipo en microorganismos.

\section{Agradecimientos}

El trabajo en el laboratorio de los autores es posible gracias a la ayuda CIVP18A3896 de la Fundación Ramón Areces. Agradecemos los comentarios críticos y apoyo de Juana M. Gancedo y las sugerencias de José M. Siverio (Facultad de Ciencias, Universidad de La Laguna). 


\section{REFERENCIAS}

Becker-Kettern, J., Paczia, N., Conrotte, J.F., Zhu, C. Fiehn, 0., Jung, P.P., Steinmetz, L.M., Linster, C.L. $\mathrm{NAD}(\mathrm{P}) \mathrm{HX}$ repair deficiency causes central metabolic perturbations in yeast and human cells. FEBS Journal 285, 3376-3401 (2018)

Bommer, G.T., Van Schaftingen, E., Veiga-da-Cunha, M. Metabolite repair enzymes control metabolic damage in glycolysis. Trends Biochem Sci.45, 228-243 (2020).

Colinas, M., Shaw, H.V., Loubéry, S., Kaufmann, M., Moulin, M., Fitzpatrick, T.B. A pathway for repair of $\mathrm{NAD}(\mathrm{P}) \mathrm{H}$ in plants. J Biol Chem. 289, 14692-14706 (2014).

Collard, F., Baldin, F., Gerin, I., Bolsée, J., Noël, G., Graff, J., Veiga-da-Cunha, M., Stroobant, V., Vertommen, D., Houddane, A., Rider, M.H., Linster, C.L., Van Schaftingen, E., Bommer, G.T. A conserved phosphatase destroys toxic glycolytic side products in mammals and yeast. Nat Chem Biol. 12 601-607 (2016)
Despotović, D., Brandis, A., Savidor, A., Levin, Y., Fumagalli, L., Tawfik, D.S. Diadenosine tetraphos phate (Ap4A) - an E. coli alarmone or a damage metabolite? FEBS J. 284, 2194-2215 (2017).

Petrovova, M., Tkadlec, J., Dvoracek, L., Streitova E., Licha, I. NAD(P)H-Hydrate Dehydratase- A metabolic repair enzyme and its role in Bacillus subtilis stress adaptation. PLoS One 9(11): e112590. https:// doi.org/10.1371/journal.pone.0112590 eCollection 2014.

Silver, P.A., Way, J.C, Arnold, F.H., Meyerowitz, J.T. Synthetic biology: Engineering explored. Nature. 509, 166-167 (2014).

Sols, A., Crane R. Substrate specificity of brain hexokinase. J Biol Chem. 210, 581-595 (1954).

Van Bergen, N.J. et al. (35 autores) NAD(P HX dehydratase (NAXD) deficiency: a novel neurodegenerative disorder exacerbated by febrile illnesses. Brain, 142, 50-58 (2019) Hay una presentación en https://www.youtube.com watch? $v=\mid \mathrm{BP} 00 \mathrm{ia} 71 \mathrm{wk}$
Veiga-da-Cunha, M., Chevalier, N., Stephenne, X., Defour, J.P., Paczia, N., Ferster, A., Achouri, Y., Dewulf, J.P., Linster, C.L., Bommer, G.T., Van Schaftingen, E. Failure to eliminate a phosphorylated glucose analog leads to neutropenia in patients with G6PT and G6PC3 deficiency. Proc Natl Acad Sci U SA. 116, 1241-1250 (2019).

Yamanouchi, T., Tachibana, Y., Akanuma, H., Minoda, S., Shinohara, T., Moromizato, H., Miyashita, H., Akaoka, I. Origin and disposal of 1,5-anhydroglucitol, a major polyol in the human body. Am J Physiol. 263, E268-E273 (1992).

Yu, J., Liu, Z., Liang, Y., Luo, F., Zhang, J., Tian, C., Motzik, A., Zheng, M., Kang, J., Zhong, G., Liu, C., Fang, P., Guo, M., Razin, E., Wang, J. Second messenger $\mathrm{Ap}_{4} \mathrm{~A}$ polymerizes target protein HINT1 to transduce signals in FceRl-activated mast cells. Nat. Commun. 10, 4664 (2019) doi.org/10.1038/s41467-019-12710-8.

Zamecnik, P. Diadenosine 5',5'"-P1,P4-tetraphosphate (Ap4A): its role in cellular metabolism. Anal. Biochem. 134, 1-10 (1983)

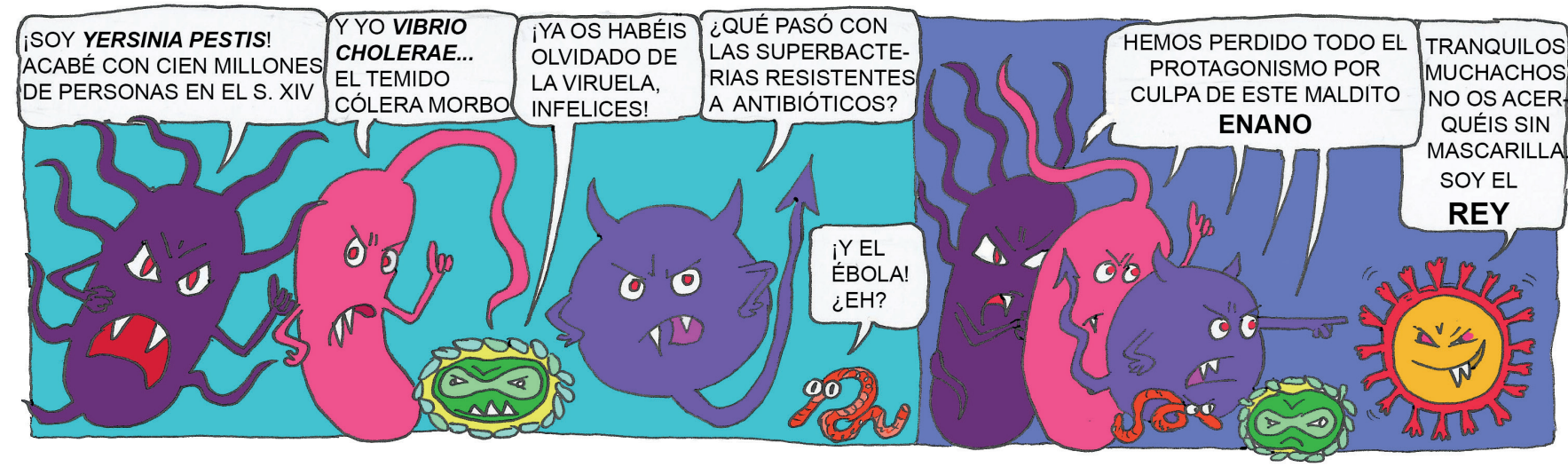

